\title{
Solid Lipid Nanoparticles: A Potential Approach for Dermal Drug Delivery
}

\author{
Pratibha G. Kakadia ${ }^{1, *}$, Barbara R. Conway \\ Department of Pharmacy, School of Applied Sciences, University of Huddersfield, Queensgate, Huddersfield HD1 3DH, UK \\ *Corresponding author: pratibha.kakadia@hud.ac.uk
}

Received August 18, 2014; Revised September 18, 2014; Accepted September 22, 2014

\begin{abstract}
Solid lipid nanoparticles (SLNs) have attracted increasing attention during recent years. Due to their unique size dependent properties, lipid nanoparticles offer possibilities to develop new therapeutics. The ability to incorporate drugs into nanoparticles offers a new prototype in drug delivery thus realizing the dual goal of both controlled release and site-specific drug delivery. Drug delivery to the skin is widely used for local and systemic delivery and has potential to be improved by application of nanoparticulate formulations. If investigated appropriately, solid lipid nanoparticles may open new opportunities in therapy of complex diseases which is difficult to treat.
\end{abstract}

Keywords: solid lipid nanoparticles, dermal delivery, colloidal carriers

Cite This Article: Pratibha G. Kakadia, and Barbara R. Conway, "Solid Lipid Nanoparticles: A Potential Approach for Dermal Drug Delivery.” American Journal of Pharmacological Sciences, vol. 2, no. 5A (2014): 1-7. doi: 10.12691/ajps-2-5A-1.

\section{Introduction}

Targeted delivery of drug molecules to specific organ sites such as the skin or eye is one of the most challenging areas of research in pharmaceutical development. The skin is the largest organ of the body and functions as a protective layer. The large surface area $\left(1.8 \mathrm{~m}^{2}\right)$ and easy accessibility of skin make it an attractive route for drug delivery. However, the unique structure of skin limits the transport of molecules through it [1]. The skin is broadly categorized into the non-viable epidermis called stratum corneum, viable epidermis, and dermis but is a complex structure with sweat glands, hair follicles and blood vessels (Figure 1).

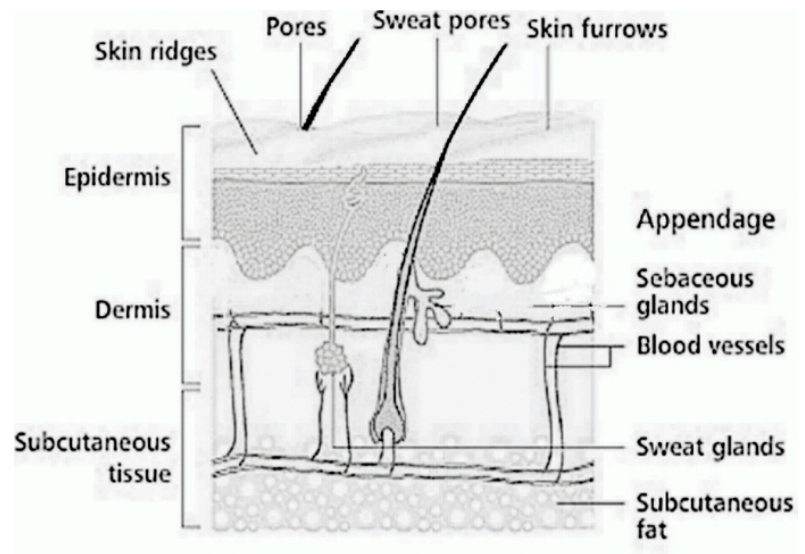

Figure 1. Structure of skin

The stratum corneum (SC), also called the horny layer, has been shown to be the main skin barrier. It is composed of closely packed keratinized cells, also called corneocytes. The intercellular spaces in SC are filled with lipid bilayers (lamellae), which are composed of non-polar lipids, including ceramides (47\%), free fatty acids (9\%) and their esters as well as cholesterol (27\%) and sulphates. The structure of the lipid bilayer demonstrates heterogeneity, providing both lipophilic and hydrophilic domains [2]. Molecules can penetrate the skin by three main routes [3] which are described as follows:

1) Intracellular transport involves drug transport through corneocytes and intervening lipids; it is the most favorable route for lipophilic compounds.

2) Intercellular transport is the movement of molecules between the lipids through aqueous regions. It is the major pathway for most drugs, in which molecules have to pass through successive hydrophilic domains in lipid bilayers.

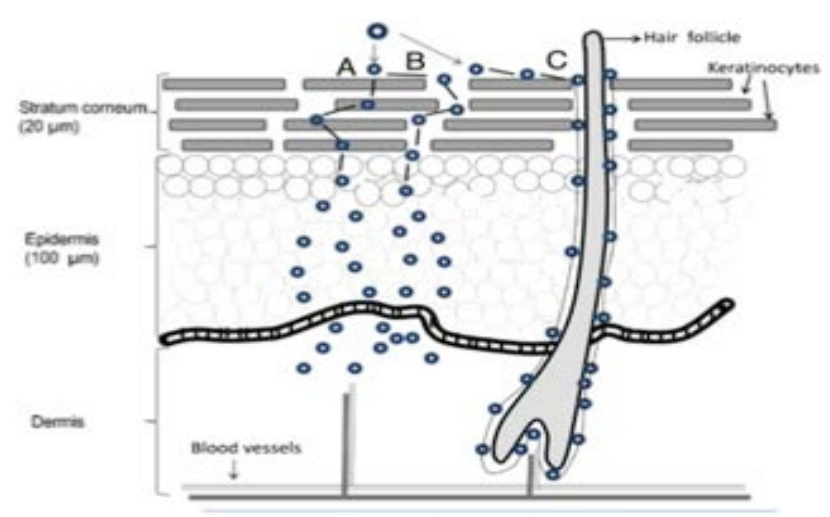

Figure 2. Schematic representation of penetration routes of drugs throughout the skin [4]

3) Skin appendageal transport describes transport across skin appendages such as hair follicles and sebaceous 
glands. It serves as a shunt pathway for iontophoretic transport of charged molecules and penetration of particulate systems.

Colloidal particles in the size range 1 to $1000 \mathrm{~nm}$ are known as nanoparticles. Nanoparticles are of great scientific interest as theyform a bridgebetween bulk materials and atomic or molecular structures [5].

Nanoparticles for drug delivery comprise drug molecules, which are dissolved, entrapped, or absorbed into a nanoparticle matrix. In recent years, significant effortshave been made to develop nanotechnology for drug delivery since it offers a suitable means of delivering low molecular weight drugs and macromolecules such as proteins, peptides or genes to cells and tissues. As a consequence of their small size, nanoparticles can penetrate through small capillaries, are taken up by cells and allow drug release at specific sites in the body for certainduration to facilitate accurate delivery. This can enhance the therapeutic effect and reduces toxic effects. This review paper focuses on use of SLNs in terms of their advantages, production methodology, characterization and their promising role in dermal delivery.

\section{Solid Lipid Nanoparticles}

Solid lipid nanoparticles (SLNs) offer an attractive means of drug delivery, particular for poorly water-soluble drugs. They were developed at the beginning of 1990s as an alternative carrier system to emulsions and liposomes. The sub-micron colloidal carriers are prepared by replacing a liquid lipid (oil) of an o/w emulsion by a physiologically biocompatible solid lipid or a blend of solid lipids, i.e. a lipid particle matrix which is solid at both room and body temperature [6,7]. A significant benefit of these formulations is that they can be prepared using excipients with generally recognized as safe (GRAS) status for oral and dermal delivery, which decreases risks of associated toxicity.

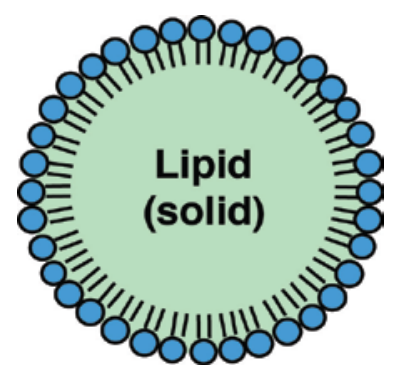

Figure 3. Structure of solid lipid nanoparticle [8]

The skin, especially the stratum corneum, behaves as a passive barrier to penetrant molecules. Penetration enhancers are substances that can facilitate the absorption of penetrant through skin by temporarily diminishing the impermeability of skin. Various permeation enhancers such as terpenes, essential oils, fatty acids and esters, alcohols, glycols, glycerides and phospholipids have been described in the literature [9].

\subsection{Advantages of SLNs}

SLNs offer a number of potential advantages over other formulations. The use of physiological and biodegradable lipids,not only decreases the risks of acute and chronic toxicity, but some of the manufacturing methods can also avoid the use of organic solvents [10]. Relatively high encapsulation efficiencies lead to reducedrequirements for active drug during the formulation process, as well as improved bioavailability of poorly water soluble molecules [11]. Obviously, like most encapsulation technologies, they can protect labile agents from degradation in the environment of the body [12]. They are a versatile formulation with relatively low associated production costs but they have an improved stability profile compared to liposomal formulations and can be freeze-dried. However, they are not without their challenges, such as particle growth over time or potential for modification of the lipid structure into a crystal lattice. This could cause expulsion of drug from lipid particles during storage [13]. They can also have an unpredictable gelation tendency [8].

\section{Method of Preparation}

\subsection{Primary Production Methods}

\subsubsection{High-Pressure Homogenization (HPH)}

$\mathrm{HPH}$ is a reliable and powerful technique, used for production of solid lipid nanoparticles. High-pressure homogenizers push liquid at high pressures $(100-2000$ bar), through a narrow gap (in the range of few microns). The fluid accelerates over a very short distance under very high shear stress and cavitation forces disrupt the particles down to the submicron range. Generally 5-10\% lipid content is used but up to $40 \%$ lipid content has also been investigated [8].

\section{A. Hot homogenization}

Hot homogenization is carried out at temperatures above the melting point of the lipid and can therefore be regarded as homogenization of an emulsion. A preemulsion of the drug-loaded lipid is melted and the aqueous emulsifier phase (maintained at the same temperature) is obtained using high shear mixing. In general, higher temperatures result in smaller particle sizes due to decreased viscosity of the inner phase. However, higher temperatures can increase the degradation rate of drug and the carrier. Increasing the homogenization pressure or the number of cycles often results in an increase in the particle size due to high kinetic energy of the particle.

B. Cold homogenization

Cold homogenization has been developed to overcome various problems associated with hot homogenization, such as temperature-induced drug degradation and uneven drug distribution within the aqueous phase during homogenization. During cold homogenization the lipid melt containing drugis rapidly cooled (e.g.by means of dry ice or liquid nitrogen). The solid, drug-containing lipid is milled to form microparticles. The solid lipid microparticles are dispersed in a cold surfactant solution forming a pre-suspension, which is subjected to homogenization at or below room temperature.

\subsubsection{Solvent Emulsification-Diffusion Method}

SLNs can also be produced by solvent emulsificationdiffusion techniques. The mean particle size depends upon 
lipid concentration in the organic phase and the emulsifier used. Particles with average diameters of 30-100 nm can be obtained by this technique. Avoidance of heat during preparation is the most important advantage of this technique. In this method, the lipid matrix is dissolved in a water-immiscible organic solvent followed by emulsification in an aqueous phase. The solvent is evaporated under reduced pressure, resulting in a nanoparticulate dispersion formed by precipitation of the lipid in aqueous medium [14].

\subsubsection{Solvent Evaporation}

SLNs can also be produced by solvent evaporation. The lipophilic material is dissolved in a water-immiscible organic solvent and is emulsified into an aqueous phase. Upon evaporation of the solvent, a nanoparticle dispersion is formed by precipitation of the lipid in the aqueous medium. The solution is emulsified in an aqueous phase by high pressure homogenization and the organic solvent removed by evaporation under reduced pressure [15].

\subsubsection{Microemulsion-based Method}

Gasco and coworkers (1997) developed SLNs based on dilution of microemulsions. These are made by heating low melting fatty acids like stearic acid just above their melting point, an emulsifier (e.g., polysorbate 20, polysorbate 60 , soyaphosphatydylcholine and taurodeoxycholic acid sodium salt), co-emulsifiers (e.g.,butanol, sodium monooctylphosphate) and water. The hot microemulsion is dispersed in cold water $\left(2-3^{\circ} \mathrm{C}\right)$ under stirring. Typical volume ratios of the hot microemulsion to cold water are in the range of $1: 25$ and 1:50. The dilution process is critically determined by the composition of the microemulsion. The SLN dispersion can be used as a granulation fluid for transferring into solid products like tablets and pellets, but in case of low particle content, large amounts of water need to be removed. Nanoparticles are produced using a solvent which distributes rapidly into the aqueous phase (e.g., acetone), while larger particle sizes can be obtained with more lipophilic solvents. The hydrophilic co-solvents in the microemulsion might play a similar role in the formation of lipid nanoparticles as the acetone doesin the formation of polymer nanoparticles [16].

\subsubsection{Spray Drying Method}

Spray drying is an alternative procedure to lyophilization in order to transform an aqueous SLN dispersion into a solid product. This method can cause particle aggregation due to high temperatures, shear forces and partial melting of the particle. Freiitas and Muller recommend the use of lipids with melting points $>70^{\circ} \mathrm{C}$ for spray drying [17].

\subsubsection{High-speed Homogenization Followed by Ultrasonication}

SLNs can also be produced by sonication or high speed stirring followed by ultrasonication. The first stepsare similar to high-pressure homogenization. A coarse emulsion is obtained using probe or bath sonication. This is then subjected to ultrasonication until the desired sized nanoemulsion is obtained. It is very simple and can be advantageous over other methods like hot and cold homogenization because the equipment used in this technique is very common. The method does however result in larger size distributions, ranging up to the micrometer range, which can lead to physical instability,including particle growth upon storage and also metal contamination due to ultrasonication[18].

\subsubsection{Double Emulsion Method}

The double emulsion (w/o/w) method is based on the solvent emulsification-evaporation method. It is mainly used for the production of lipid nanoparticles loaded with hydrophilic drugs. In this case, the drug and emulsifier are encapsulated in the inner aqueous phase of w/o/w double emulsion. A stabilizer is required to prevent drug partitioning into the outer aqueous phase during solvent evaporation. These types of formulation are often referred to as lipospheres due to their comparatively larger particle size compared to SLNs [19].

\subsubsection{Supercritical Fluid Method}

This is an alternative method of preparing SLNs using particles from gas-saturated solutions (PGSS). This technique has several advantages such as (i) avoiding the use of solvents; (ii) particles are obtained as a dry powder, instead of suspension; (iii) it required mild pressure and temperature conditions. Carbon dioxide solution is a good choice as a solvent for this method [20].

\subsubsection{Precipitation Method}

The lipid is dissolved in an organic solvent (e.g., chloroform) and the solution is emulsified into an aqueous phase. After evaporation of the organic solvent, the lipid is precipitated, forming nanoparticles [21].

\subsubsection{Film-ultrasound Dispersion}

The lipid and drug are added to suitable organic solutions, and after decompression, rotation and evaporation of the organic solutions, a lipid film is formed. The aqueous solution containing emulsifier is then added to lipid film and, using probe sonication, SLNsare formed. Oleanolic acid SLNshave been produced using soybean phospholipid as a carrier using the film-ultrasound technique [13].

\subsubsection{Melting Dispersion Technique}

In the melting dispersion technique, drug and solid lipid are melted in an organic solvent and an oil phase is added slowly into a small volume of water (preheated to the same temperature), with continuous stirring at high rates for few hours. It is then cooled to room temperature to give SLNs. Reproducibility is better than ultra-sonication methods but less than that of solvent-emulsification evaporation methods [22].

\subsubsection{Membrane Contractor Technique}

In the membrane contractor technique, the liquid phase is forced, at a temperature above the melting point of the lipid, through the pores in a membrane to form small droplets. The advantage of this technique is its ease of use and the control of the particle size by suitable choice of process parameters. The aqueous phase is stirred continuously and circulates tangentially inside the membrane module, which can be temperature controlled, 
and sweeps away the droplets being formed at the pore outlets. Upon on cooling the formulation to room temperature, SLNs are formed. This method may be reasonably straightforward to scale-up [23].

\subsection{Secondary Production Methods}

\subsubsection{Freeze-drying}

Water can be removed in order to improve physical and chemical stability of these systems. Freeze-drying is the most commonly used process in the pharmaceutical field for conversion of solutions or suspensions into solids of sufficient stability for distribution and storage. Freezedrying, also known as lyophilization, is an industrially scalable process, which consists of removing of frozen water by sublimation, and desorption under vacuum. Lyophilization has been used to achieve long stability durations for products containing hydrolysable drugs or a suitable product for per-oral administration.

For all the lipid matricesstudied, freeze-drying leads to the formation of larger SLNs, with a wider size distribution, due to presence of aggregates between the nanoparticles. An adequate amount of cryoprotectant can protect the aggregation of SLNs during the freeze-drying process [24].

\subsubsection{Sterilization}

For parenteral administration, SLNs dispersions must be sterile. Aseptic production, filtration, gamma irradiation and autoclaving are commonly used to achieve sterilization. Aseptic conditions can be used during production of sterile SLNs but requirements can be complex and expensive. Sterilization by autoclaving is popular and convenient but it has some disadvantages; the high temperaturesencountered during autoclaving can cause coalescence, as there is no applied shear to prevent this. Gamma irradiation does have some significant advantages over other methods such as better assurance of product sterility than filtration and aseptic processing [25].

\section{Characterization of SLNs}

Characterization of the SLNs is necessary for product development and quality but presents serious challenges due to colloidal size of particles and complexity and dynamic nature of delivery system.

\subsection{Measurement of Particle Size and Zeta Potential}

Particle size analysis can be performed by photon correlation spectroscopy (PCS), laser diffractometry (LD) and Nanoparticle tracking analysis (NTA). PCS (also known as dynamic light scattering) measures the fluctuation of intensity of the scattered light which is caused by particle movement [26]. This method can measure particle size ranging from few nanometers to about 3 microns. The LD method is based on the dependence of the diffraction angle on the particle size and it is useful for size ranges from $100 \mathrm{~nm}$ to $180 \mu \mathrm{m}$.

NTA is a relatively new method of visualising and analysing particles from $10-1000 \mathrm{~nm}$ in liquids. It measures particle size based on rate of Brownian motion, which is related to the viscosity of the liquid, the temperature and the size of the particle [27,28].

Zeta potential measurement, an indicator of the stability of colloidal dispersions, can be determined using a zeta potential analyzer. Before measurement, SLNs dispersions are diluted appropriately, often with deionized water, to measure zeta potential. The surface charge will reflect the type of lipid used in the formulation and can be used to inform long-term predictions about the storage stability of colloidal dispersions [29].

\subsection{Determination of Drug Loading and Entrapment Efficiency}

It is of primary importance to determine the amount of drug incorporated in SLNs, since this influences release characteristics and the feasibility of the formulation in terms of amount of formulated product to be delivered. The degree of encapsulation can be assessed ultimately by determining the quantity of drug remaining in the supernatant after centrifugation of a SLN suspension or, alternatively, by dissolution of the sediment in a suitable solvent and subsequent analysis. Standard analytical techniques such as spectrophotometry orhigh performance liquid chromatography can be used to assay the drug [30].

\subsection{Electron Microscopy}

Scanning electron microscopy (SEM) and transmission electron microscopy (TEM) are direct methods used for morphological examination of nanoparticles. TEM has a lower size limit of detection [31].

\subsection{Atomic Force Microscopy (AFM)}

In this technique, a probe tip with atomic scale sharpness is rastered across the sample to produce a topological map based on the forces at play between the tip and surface. The probe can be dragged across sample (contact mode), or allowed to hover just above (noncontact mode), with the exact nature of the particular force employed serving to distinguish among the sub-techniques. A high resolution is obtainable with this approach, which along with the ability to map a sample according to properties in addition to size, e.g., colloidal attraction or resistance to deformation, makes AFM a valuable tool [32].

\subsection{Structural Analysis}

Among a large number of analytical techniques, differential scanning calorimetry (DSC) and powder x-ray diffraction (XRD) can be used to elucidate structural information on the dispersed drug and lipids. Crystallinity of drug and excipients can be measured using XRD by scattering of radiation from crystal plane within the solid, while DSC can be used to determine nature of crystallinity within nanoparticles through the measurement of glass transitions and melting point temperatures and their enthalpies [8].

\subsection{Nuclear Magnetic Resonance (NMR)}

High-resolution NMR can be used to determine both the size and the qualitative nature of nanoparticles. The selectivity afforded by chemical shifts compliments the 
sensitivity to molecular mobility, thus providing information on the physicochemical status of components within the nanoparticle [33].

\subsection{In-vitro Drug Release from SLNs}

SLN dispersions can be placed in prewashed dialysis tubing, which can be hermetically sealed. The dialysis sac is then dialyzed within an appropriate dissolution medium at(37 \pm 0.5$)^{\circ} \mathrm{C}$. Samples are withdrawn at suitable intervals from the dissolution medium, centrifuged and analyzed for drug content using an appropriate analytical method [34,35].

\section{Nanoparticles for Dermal Delivery}

Although topical delivery of compounds to the skin has been common throughout history, it is only since 1970s, with the advent of transdermal patches, that it has been used widely as a route for systemic delivery. Different carrier systems have been proposed in an attempt to enhance the transport of drugs through the skin, enabling drug retention and in some cases allowing a controlled release. Effective skin penetration has many applications, for example, drug delivery to skin (dermatological treatments) and through skin (transdermal treatments), and skin care and protection (cosmetics). Potential sites for nanoparticle dermal delivery include the skin surface, furrows and hair follicles.

The hair follicle is a tube-like pocket of the epidermis that extends through most of the depth of the skin and encloses a small papilla of dermis at base. Microparticulate vesicles, like liposomes and nanoparticles have been shown to deliver drug molecules much deeper into hair follicles than conventional formulations like creams and ointments. It has been shown that nanoparticles between $300 \mathrm{~nm}$ and $750 \mathrm{~nm}$ penetrate preferentially into hair follicles. Other studies show titanium dioxide particles, about $100 \mathrm{~nm}$ in diameter, penetrating into the hair follicle [36].

SLNs offer an occlusive effect due to film formation on the skin surface, which reduces transepidermal water loss. Occlusion also favors drug penetration into the skin. The high specific area of nanometer-sized SLNs enhances contact of encapsulated drug with the stratum corneum [37]. Furthermore lipid nanoparticles are able to enhance the chemical stability of compounds sensitive to light, oxidation and hydrolysis.

\subsection{Application of Nanocarrier Systems in Dermal Delivery}

Topical treatment of skin conditions has the advantage that high drug levels can be achieved at the site of disease and systemic side effects can be reduced compared to oral or parenteral drug administration. Topical drug administration is a challenge in pharmaceutics due to difficulties in controlling and determining the exact amount of drug that reaches the different skin layers. The drugs' and the vehicles' physicochemical properties are considered to be main determinants responsible for the drug differential distribution in the skin. Lipid nanoparticles have been investigated to improve the treatment of skin disease such as atopic eczema, psoriasis, acne, skin mycosis and inflammation. Apart from the treatment of the skin diseases by topical application, gastrointestinal side effects of non-steroidal anti-inflammatory drugs can be decreased by topical antirheumatictherapy, for example [38].

SLNs have been shown to sustain the delivery of sunscreens in human studies and that the results correlate well with in-vitro penetration studies[39]. Nanoparticle preparations are also currently under investigation for novel treatment of dermatological conditions such as acne vulgaris, recurrent condylomaaccuminata, atopic dermatitis, and hyperpigmented skin lesions [40].

\subsubsection{Condyloma Acuminate Treatment}

The most commonly sexually transmitted infections seen by dermatologists are due to mucosal human papillomavirus (HPV) infections. Podophyllotoxin (POD) has been shown to decrease HPV-infected epithelial cell growth, delaying the development of condyloma, acuminate but has been linked to severe skin irritation after systemic absorption. Research has shown that PODloaded SLNs may avoid systemic uptake, with preferential epidermal localization, unlike the ordinary topical cream and tincture preparations of POD. POD-loaded SLNs increased the accumulation of POD in the stratum corneum of porcine skin to nearly 3.5 times that of a $0.15 \%$ tincture. This was thought to be due to small diameters of the SLN and the enhancement of SC permeability by the soybean-lecithin incorporated into the SLN preparation [41,42].

\subsubsection{Skin Hyperpigmentation Treatment}

Melasma, post-inflammatory hyperpigmentation, results from an overproduction of melanin by melanocytes and is typically treated by sun avoidance, sunscreen, and a combination of topical all-trans retinoic acid (atRA) with hydroquinone. Although it is an effective treatment, it causes irritation and erythema and has poor stability in heat, air and light. Nano-atRA preparations can have a colloidal structure and the solid matrix of SLNs offers protection from hydrolysis and improved permeation into the stratum corneum [38].

\subsubsection{Atopic Dermatitis Treatment}

The prevalence of atopic dermatitis is increasing in industrialized countries. Acute, sub-acute and chronic atopic dermatitis are effectively managed by topical glucocorticoids. However prolonged use of glucocorticoids has been shown to cause osteoporosis, pituitary-adrenal axis suppression, growth retardation in children, and inhibition of fibroblasts leading to skin atrophy. In one study, the topical glucocorticoid, prednicarbate, was incorporated into SLNs and found to induce a localized effect in the epidermal layer particularly pronounced at 6 hours, exceeding that of standard cream and ointment nearly fourfold [43].

\subsubsection{Rheumatoid Arthritis Treatment}

Non-steroidal anti-inflammatory drugs like ketoprofen, naproxen and celecoxib,are used for the treatment of mucoskeletal disorders, such as rheumatoid arthritis, osteoarthritis. Percutaneous absorption of ketoprofenloaded SLNs incorporated into a gel was studied in-vitro. 
Also, in-vivo active localization in the stratum corneum and anti-inflammatory effects were compared to ketoprofen solution as a reference. SLNs were able to reduce drug penetration through excised human skin while it was found by tape striping, that the drug permeation and drug accumulation in the horny layer was increased. Furthermore, a prolonged anti-inflammatory effect could be shown for drug loaded SLNs compared to drug solution [44]. The anti-inflammatory activity of the diterpenoid epoxide, tiptolide was reported to be higher in a topical SLN formulation compared to a microemulsion formulation [45].

\section{Conclusion}

Solid lipid nanoparticles are a very well tolerated carrier systems for dermal application and good prospect for successful product development. It has been shown already, for various drugs, that dermal formulations containing nanoparticles can enhance penetration into the skin, increasing treatment efficiency, targeting the epidermis or follicles and reducing side effects. Advances with regards to materials and fabrication methods will facilitate the development of new nanoparticles. This review concentrates on the development of solid lipid nanoparticles and their various applications in dermal delivery. In summary, SLNs are complex systems with clear advantages and disadvantages compared to other colloidal carriers. Further work needs to be carried outto understand the structure and dynamics of SLNs at the molecular level in in-vitro and in-vivo studies.

\section{References}

[1] Kaur, P.L., Guleri, T.K., "Topical gel: a recent approach for novel drug delivery". Asia Journal of Biomedical \& Pharmaceutical Sciences, 3(17). 1-5. 2013.

[2] Haftek, M., Teillon, M. H., and Schmitt, D., "Stratum corneum, corneodesmosomes and ex vivo percutaneous penetration". Microscopy Research and Technique, 43(3). 242-249. 1998.

[3] Cevc, G. and U. Vierl, "Nanotechnology and the transdermal route: A state of the art review and critical appraisal". Journal of Controlled Release, 141(3). 277-299. 2010.

[4] Valenzuela, P., Simon, J.A., "Nanoparticle delivery for transdermal HRT".Nanomedicine: NBM, 8. S83-S89. 2012.

[5] Aryane, M.S., N. Sultana, and N. Sabah, "Fabrication of solid lipid nanoparticles for drug delivery". Pakistan Journal of Pharmaceutical Sciences, 20(3). 251-259. 2007.

[6] Pardeike, J., A. Hommoss, and R.H. Müller, "Lipid nanoparticles (SLN, NLC) in cosmetic and pharmaceutical dermal products". International Journal of Pharmceutics, 366. 170-184. 2009.

[7] Chimmiri, P., et al., "Solid lipid nanoparticles: a novel carrier for cancer therapy".International Journal of Biological and Pharmaceutical Research, 3(3). 405-413. 2012.

[8] Ekambaram, P., et al., "Solid lipid nanoparticles: a review".Scientific Reviews and Chemical Communications, 2(1). 80-102. 2012.

[9] Sinha, V.R. and M. Kaur, "Permeation enhancers for transdermal drug delivery".Drug Development and Industrial Pharmacy, 26(11). 1131-1140. 2000.

[10] Rupenganta, A., et al., "Solid lipid nanoaprticles- a versatile carrier system". Journal of Pharmacy Research, 4(7). 2069-2075. 2011.

[11] Fahr, A. and X. Liu, "Drug delivery strategies for poorly water soluble drugs". Expert Opinion on Drug Delivery, 4(4). 403-416. 2007.

[12] Garud, A., D. Singh, and N. Garud, "Solid lipid nanoparticles: method, characterization and applications". Inernational Current Pharmaceutical Journal, 1(11). 384-393. 2012.
[13] Kamble, M.S., et al., "Solid lipid nanoparticles and nanostructured lipid carriers - an overview". International Journal of Pharmaceutical, Chemical and and Biological Sciences,2(4). 681691. 2012.

[14] Trotta, M., F. Debernardi, and O. Caputo, "Preparation of Solid lipid nanoparticles by a solvent emulsification-diffusion technique".International Journal of Pharmaceutics, 257. 153-160. 2003.

[15] Yadav, N., S. Khatak, and S. Sara, "Solid lipid nanoparticles: a review".International Journal of Applied Pharmaceutics, 5(2). 818. 2013.

[16] De Labouret, A., et al., "Application of an original process for obtaining colloidal dispersion of coating polymers- Preparation, Charcterization, Industrial scaling up". Drug Development Industrial Pharmacy, 21. 229-241. 1995.

[17] Freitas, C. and R.H. Müller, "Spray drying of solid lipid nanoparticles". European Journal of Pharmaceutics and Biopharmaceutics, 46. 145-151. 1998.

[18] Patel, D., et al., "Nanostructured lipid carriers (NLC)-based gel for the topical delivery of aceclofenac: preparation, characterization, and in vivo evaluation". Scientia Pharmaceutica, 80(3). 749-764. 2012.

[19] Das, S. and A. Chaudhury, "Recent advances in lipid nanoparticle formulations with solid matrix for oral drug delivery". American Association of Pharmaceutical Scientists PharmSciTech, 12(1). 62-67. 2011.

[20] Waghmare, A.S., et al., "Solid lipid nanoparticles: a promising drug delivery". Inernational Research Journal of Pharmacy, 3(4). 100-107. 2012.

[21] Sinha, V.R., et al., "Solid lipid nanoparticles (SLN'S) - trends and implications in drug targeting". International Journal of Advances in Pharmaceutical Sciences, 1. 12-238. 2010.

[22] Reithmeier,H., J. Herrmann, and A. Gopferich, "Development and characterization of lipid microparticles as a drug carrier for somatostatin". International Journal of Pharmaceutics, 218. 133143. 2001.

[23] Charcosset, C., A. EI-Harati, and H. Fessi, "Preparation of solid lipid nanoparticles using a membrane contactor". Journal of Controlled Release, 108. 112-120. 2005.

[24] Abdelwahed, W., et al., "Freeze-drying of nanoparticles: formulation, process and storage considerations". Advanced Drug Delievery Review, 58(15). 1688-713. 2006.

[25] Loxley, A., "Solid lipid nanoparticles for delivery of pharmaceutical active". Drug Delivery Technology, 9(8). 2009.

[26] Wolfgang, M. and K. Madar, "Solid lipid nanoparticles production, characterization and applications". Advanced Drug Delivery Review, 47. 165-196. 2001.

[27] Kakadia, P., et al. "Topical drug delivery of nanoencapsulated triclosan". in $41^{\text {st }}$ Annual Meeting \& Exposition of the Controlled Release Society. Chicago.

[28] Moddaresi, M., et al., "The role of vehicle-nanoparticle interactions in topical deug delivery".International Journal of Pharmaceutics, 400(1-2). 176-182. 2010.

[29] Luo, Y., et al., "Solid lipid nanoparticles for enhancing vinpocetine's oral bioavailability". Journal of Controlled Release, 114. 53-59. 2006.

[30] Nair, R., et al., "Recent advances in solid lipid nanoparticle based drug delivery systems".Journal of Biomedical Sciences and Research, 3(2). 368-384. 2011.

[31] Kamble, V.A., D.M. Jagadle, and V.J. Kadam, "Solid lipid nanoparticles as drug delivery system".International Journal of Pharmaceutics and Biopharmaceutics, 1(3). 1-9. 2010.

[32] Mukherjee, S., S. Ray, and R.S. Thakur, "Solid lipid nanoparticles (SLN): a modern formulation approach in drug delivery system".Indian Journal of Pharmaceutical Sciences, 71(4). 349358. 2009.

[33] Gomez, M.V., et al., "Nanoparticles size determination by ${ }^{1} \mathrm{H}$ NMR spectroscopy".Journal of the Americal Chemical Society, 131(41). 14634-14635. 2009.

[34] Verma, V.K. and A. Ram, "Preparation, characterization and invitro release of piroxicam-loaded solid lipid nanoparticles". International Journal of Pharmaceutical Sciences and Nanotechnology, 3(3). 1136-1143. 2010.

[35] Wang, J.W., et al., "Preparation and quality evaluation of oleanolic acid-loaded solid lipid nanoparticles". Journal of Fourth Military Medical University, 28. 472-472. 2007. 
[36] Konrádsdóttir, F., et al., "Drug targeting to the hair follicles: a cyclodextrin-based drug delivery". American Association of Pharmaceutical Scientists PharmSciTech, 10(1). 266-269. 2009.

[37] Jenning, V., et al., "Vitamin A loaded solid lipid nanopartilces for topical use: occlusive properties and drug targeting to the upper skin". European Journal of Pharmaceutics and Biopharmaceutics, 49. 211-218. 2000

[38] Schäfer-Korting, M., M. Wolfgang, and H. Korting, "Lipid nanoparticles for improved topical application of drugs for skin diseases". Advanced Drug Delivery Review, 59. 427-443. 2007.

[39] Wissing S.A., M.R.H., "Solid lipid nanoparticles as carrier for sunscreens: in vitro release and in vivo skin penetration". Journal of Controlled Release,81. 153-160. 2002.

[40] Wiesenthal, A., et al., "Nanoparticles: small and mighty". The International Journal of Dermatology, 50. 247 - 254. 2011.
[41] Chena, H., et al., "Podophyllotoxin-loaded solid lipid nanoparticles for epidermal targeting". Journal of Controlled Release, 110(2). 296-306. 2006.

[42] Wolff, K. and R.A. Johnson, "Fitzpatrick's Color Atlas and Synopsis of Clinical Dermatology, 6th edn. Part III-Diseases due to microbial agents". 2009, New York: McGraw-Hill.

[43] Maia, C.S., W. Mehnert, and M. Schaller, "Drug targeting by solid lipid nanoparticles for dermal use". Journal of Drug Targeting, 10. 489-495. 2002.

[44] Puglia, C., et al., "Lipid nanoparticles for prolonged topical delivery: an invitro and in vivo investigation". International Journal of Pharmaceutics, 357. 295-304. 2008.

[45] Mei, X., et al., "Comparison of toxicokinetic and tissue distribituion of triptolide-loaded solid lipid nanoparticles vs free triptolide in rates". European Journal of Pharmaceutical Sciences, 47. 713-717. 2012. 